\title{
Top-down predictive impairment may play a potential role in social cognition in patients with CRPS
}

\author{
Dasom Lee ${ }^{1,2}$, Do-Hyung Kang ${ }^{1}$ \\ ${ }^{1}$ Emotional Information and Communication Technology Association, Seoul, Republic of Korea; ${ }^{2}$ Department of Psychiatry, Seoul National \\ University Hospital, Seoul, Republic of Korea \\ Correspondence to: Do-Hyung Kang, MD, PhD. Emotional Information and Communication Technology Association, 508, Samseong-ro, Gangnam- \\ gu, Seoul, Republic of Korea. Email: basuare@hanmail.net. \\ Comment on: Brown CA, Scholtes I, Shenker N, et al. Suboptimal learning of tactile-spatial predictions in patients with complex regional pain \\ syndrome. Pain 2020;161:369-78.
}

Submitted Apr 09, 2020. Accepted for publication Sep 08, 2020.

doi: 10.21037/apm-20-900

View this article at: http://dx.doi.org/10.21037/apm-20-900

In the February issue of Pain, Brown et al. reported their investigation into the potential cognitive mechanism underlying tactile sensory deficits in patients with complex regional pain syndrome (CRPS) (1). Their study was based on the hierarchical predictive coding (HPC) model of perception: sensory inputs can be predicted and explained by higher-order probability-related knowledge. They used a tactile-spatial oddball task and hierarchical Bayesian model for predictive coding, hypothesizing that statistical learning of the prediction of sensory change would be suboptimal in patients with CRPS. Their results demonstrated that the patients' predictions were less precise and less finely tuned to the statistics of the environment, providing evidence of top-down predictive impairment in patients with CRPS.

The main strength of this study is that it was the first study to use a generative mechanistic model to investigate tactile perception in chronic pain disorders. Tactile sensory deficits in chronic pain conditions have been studied using different tasks that measure the ability to discriminate, identify, or localize tactile stimuli (2). Previous findings revealed that tactile spatial acuity was decreased in different pain conditions, including hand dystonia, chronic low back pain, arthritis, and CRPS $(2,3)$. Moreover, this tactile impairment is known to be associated with the reorganization of the primary and secondary somatosensory cortex; the reduced hemodynamic signal during electrical stimulation of the index fingers of the affected hand was linked to impaired tactile discrimination and individual pain intensity in patients with CRPS (4).
The tactile spatial oddball task was first validated by the study conducted by Brown et al. In the oddball paradigm, digital ring electrode pairs were placed on each digit of the patients' fingers. Patients were required to respond if they detected a shift in electrical stimulation from one digit to another. The probability of tactile spatial change varies across different blocks such that oddballs could occur with a probability of $10 \%, 30 \%$, or $50 \%$. From the theoretical perspective of HPC, unpredicted and surprising sensory inputs (i.e., the occurrence of oddball) are propagated as prediction errors from low- to high-level cortical regions. Neurophysiological studies have shown that tactile awareness relies on recurrent neural processing (feedback - feedforward loop) within the somatosensory network (5), and mismatched responses to tactile stimuli reflect hierarchical Bayesian inference in somatosensory and limbic areas in a roving tactile oddball task (6). In addition, a recent neuroimaging study revealed that unexpected and surprising tactile changes increase the connectivity within a caudal-torostral ascending hierarchy of somatosensory, limbic, and prefrontal areas, and that hierarchical processing of tactile prediction error may be coordinated by anterior insula (7).

Furthermore, the authors modeled and simulated the manner in which the statistical structure of the environment might be internally represented and updated, by applying hierarchical Bayesian inference and a computational model of predictive coding to a tactile-spatial change-detection task. From the perspective of the Bayesian model, the participants' estimation of the probability of a change in the 
Table 1 Impaired executive function in chronic pain condition

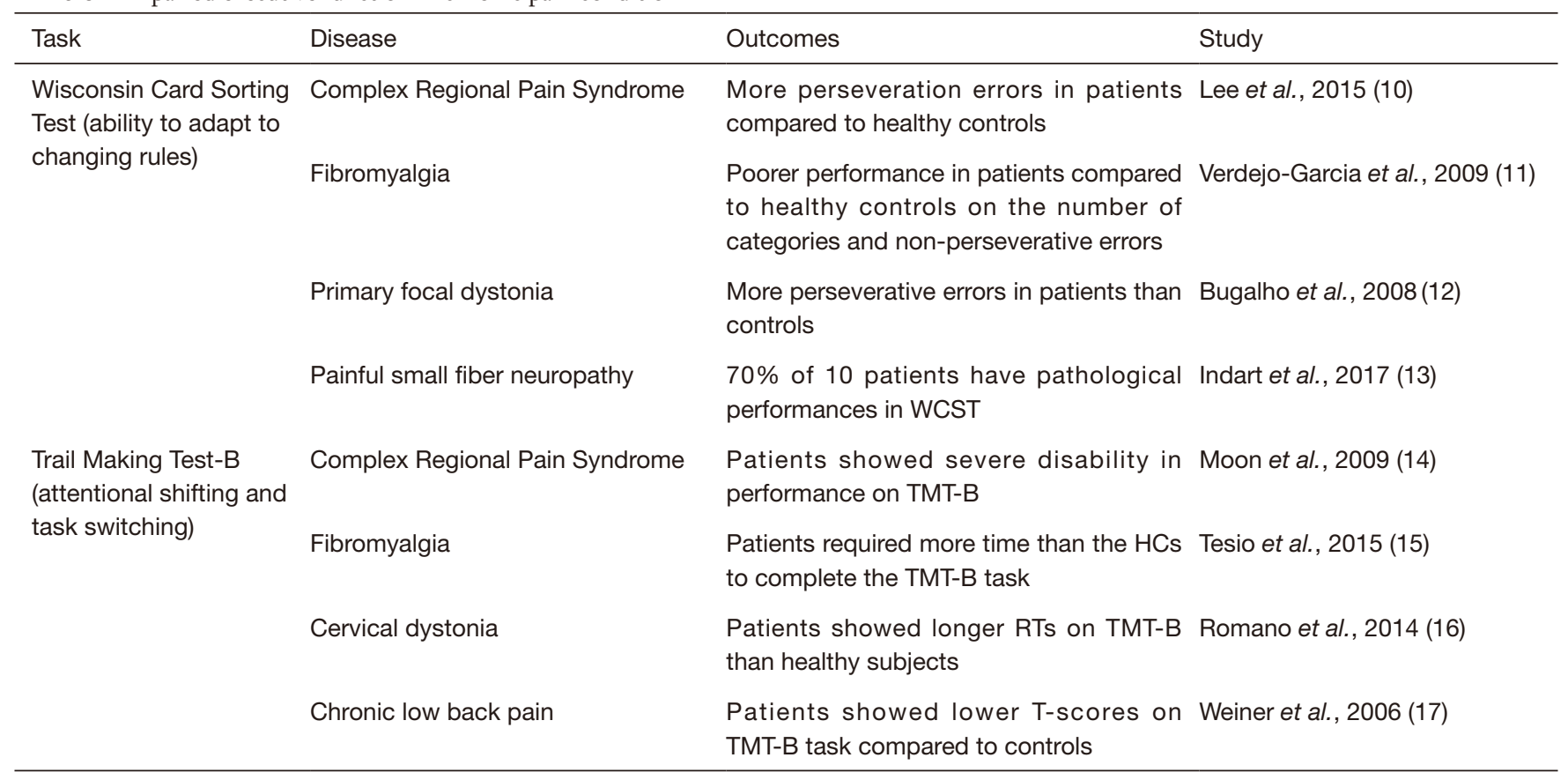

location of the stimulus are not fixed but constantly updated with time, according to the prediction errors and their precision weights. Thus, trial-by-trial analysis can provide information on history-dependent statistical learning, which can be ignored in condition-averaged data. This trial-wise approach provides significant methodological advances, compared to earlier research, and such an approach to the investigation into the role of higher-order statistical learning in perceptual disturbances in CRPS is highly interesting. This novel and advanced methodology is expected to contribute to a better understanding of the pathophysiological mechanisms underlying CRPS.

Their study reveals some insights into establishing a therapeutic strategy for CRPS. Sensory retraining involves simple tasks such as tactile discrimination, but it does not aim to enhance adaptive, flexible top-down learning. It is well known that patients with cognitive impairments are less able to communicate clearly about their pain. In effect, decreased cognitive flexibility was shown to negatively affects the ability to use cognitive restructuring for the treatment of anxiety, although diminished cognitive flexibility does not necessarily prevent patients from benefitting from cognitive behavioral therapy (8). Individual cognitive function may be a useful clinical marker to help establish therapeutic strategy and predict the treatment efficacy for patients, though more evidence is required to consider this cognitive mechanism in the treatment of pain.

As the authors stated, statistical learning allows individuals to make predictions and facilitates adaptive behavior. The ability to extract patterns or regularities from constantly changing internal sensations or the environment is important while living with chronic pain, which largely depends on an individual's cognitive function. Impaired cognitive ability has been evaluated by measuring executive function, which supports the capacity to regulate thoughts, feelings, and behavior (9). As executive functions largely coordinated by the prefrontal cortex (PFC), damage to the PFC is known to result in deficits in organizing and executing appropriate behavior, such as perseveration, poor judgment, interference with problem solving, and disinhibition. Our previous study also demonstrated the frontal abnormalities associated with CRPS (10); we observed cortical thinning in the PFC, especially in the dorsolateral and ventromedial PFC, in patients with CRPS.

Patients with other chronic pain disorders consistently exhibit impairments in cognitive flexibility, which is required for higher-level knowledge. For example, the ability to adapt to and utilize constantly changing rules, which are measured by the Wisconsin Card Sorting Test (WCST), has been found to be impaired in patients with a variety of pain conditions (Table 1). Moreover, patients with numerous pain conditions perform poorly on the Trail Making Test-B 


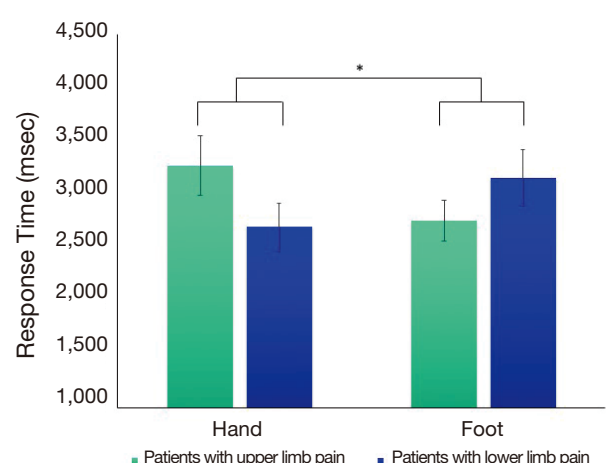

Figure 1 Differences in performance on mental rotation of hand and foot stimuli between CRPS patients with upper vs. lower limb pain (unpublished data, Lee \& Kang). Analyses were conducted on $\log$ RTs. CRPS, complex regional pain syndrome. * $\mathrm{P}<0.05$.

(TMT-B), which is known to reflect attentional shifting and task switching (Table 1). The WCST and TMT-B are used frequently to evaluate executive function. They are known to mainly map into the dorsolateral PFC, which is consistent with the notion that chronic pain may affect the PFC. Therefore, these findings provide additional evidence of impaired executive functions in several pain conditions, which may be associated with higher-level predictive impairment in patients with chronic pain.

Moreover, our recent unpublished study found impaired mental rotation ability in patients with CRPS. When presented with a picture of the hand or foot and required to judge its laterality (left or right), their performance was selectively impaired, according to their bodily state: patients with upper limb pain showed slower responses to hand stimuli than foot stimuli, while patients with lower limb pain showed the opposite pattern (Figure 1). Body representation involves different sources of top-down and bottom-up information. Our findings may imply that prior knowledge learnt from persistent pain in the affected body part may hinder patients' performance on mentally rotating the corresponding stimuli. Given that thinking about movement increase pain and swelling in patients with CRPS (18) which may be modulated by beliefs about pain and movement, it is possible that pain expectation negatively affects top-down processing of hand and foot stimuli. These findings augment and support the role of top-down processing in perception as in Brown et al. (1).

Recent views propose that predictive mechanisms may play a potential role in social cognition such as theory of mind (ToM), which inherently requires predictive skills (19).
Our previous study found potential difficulties in social interaction in CRPS; patients were less accurate in inferring another person's emotional and mental state from eye expressions compared to healthy controls (20) and exhibited a lower tendency of perspective-taking, i.e., the ability to view something from the psychological point of view of others (21). Deficits in the ToM might impair mental representation of emotions, which in turn leads to somatoform symptoms $(22,23)$. Moreover, patients with CPRS showed significantly lower empathic concern, feelings of sympathy and concern for others, compared to healthy controls (21). In addition, the level of empathic concern was associated with the severity of pain. Impaired social prediction in chronic pain has not been investigated yet. Further research is needed with individuals with chronic pain to understand the mechanism by which these factors may affect the progress and prognosis of the disease.

In conclusion, this study, with a novel theoretical approach and advanced statistical method, contributes to a more integrated understanding of the pathophysiological mechanisms underlying perceptual deficits in CRPS. However, as the authors stated, their results do not specify a somatosensory-specific deficit in patients with CRPS. First, although a novel tactile-spatial change-detection task was employed in their study, the abnormality of the top-down neurocognitive function in CRPS has already been robustly reported. Second, considering the abnormality in the insula and its connection to multiple brain regions in the neural network of perception, there may be some statistical difficulties in distinguishing the somatotopic abnormality of the insula from the somatosensory components involved in interoception and body schema. The existing data are not sufficient to confirm whether Brown et al.'s results can be solely attributed to a somatosensory-specific deficit in CRPS. Future studies should expand the discussion on the neurocognitive mechanisms underlying the perceptual disturbances in CRPS.

\section{Acknowledgments}

Funding: This work was supported by the National Research Foundation of Korea (NRF) grant funded by the Korea government (MSIT) (No. NRF-2018R1A2B6001806).

\section{Footnote}

Provenance and Peer Review: This article was commissioned by the editorial office, Annals of Palliative Medicine. The 
article did not undergo external peer review.

Conflicts of Interest: Both authors have completed the ICMJE uniform disclosure form (available at http://dx.doi. org/10.21037/apm-20-900). The authors have no conflicts of interest to declare.

Ethical Statement: The authors are accountable for all aspects of the work in ensuring that questions related to the accuracy or integrity of any part of the work are appropriately investigated and resolved.

Open Access Statement: This is an Open Access article distributed in accordance with the Creative Commons Attribution-NonCommercial-NoDerivs 4.0 International License (CC BY-NC-ND 4.0), which permits the noncommercial replication and distribution of the article with the strict proviso that no changes or edits are made and the original work is properly cited (including links to both the formal publication through the relevant DOI and the license). See: https://creativecommons.org/licenses/by-nc-nd/4.0/.

\section{References}

1. Brown CA, Scholtes I, Shenker N, et al. Suboptimal learning of tactile-spatial predictions in patients with complex regional pain syndrome. Pain 2020;161:369-78.

2. Catley MJ, O'Connell NE, Berryman C, et al. Is tactile acuity altered in people with chronic pain? a systematic review and meta-analysis. J Pain 2014;15:985-1000.

3. Bara-Jimenez W, Shelton P, Hallett M. Spatial discrimination is abnormal in focal hand dystonia. Neurology 2000;55:1869-73.

4. Pleger B, Ragert P, Schwenkreis P, et al. Patterns of cortical reorganization parallel impaired tactile discrimination and pain intensity in complex regional pain syndrome. Neuroimage 2006;32:503-10.

5. Auksztulewicz R, Spitzer B, Blankenburg F. Recurrent neural processing and somatosensory awareness. J Neurosci 2012;32:799-805.

6. Ostwald D, Spitzer B, Guggenmos M, et al. Evidence for neural encoding of Bayesian surprise in human somatosensation. Neuroimage 2012;62:177-88.

7. Allen M, Fardo F, Dietz MJ, et al. Anterior insula coordinates hierarchical processing of tactile mismatch responses. Neuroimage 2016;127:34-43.

8. Johnco C, Wuthrich VM, Rapee RM. The influence of cognitive flexibility on treatment outcome and cognitive restructuring skill acquisition during cognitive behavioural treatment for anxiety and depression in older adults: Results of a pilot study. Behav Res Ther 2014;57:55-64.

9. Solberg Nes L, Roach AR, Segerstrom SC. Executive functions, self-regulation, and chronic pain: a review. Ann Behav Med 2009;37:173-83.

10. Lee DH, Lee KJ, Cho KI, et al. Brain alterations and neurocognitive dysfunction in patients with complex regional pain syndrome. J Pain 2015;16:580-6.

11. Verdejo-García A, Lopez-Torrecillas F, Calandre EP, et al. Executive function and decision-making in women with fibromyalgia. Arch Clin Neuropsychol 2009;24:113-22.

12. Bugalho P, Correa B, Guimaraes J, et al. Set-shifting and behavioral dysfunction in primary focal dystonia. Mov Disord 2008;23:200-6.

13. Indart S, Hugon J, Guillausseau PJ, et al. Impact of pain on cognitive functions in primary Sjogren syndrome with small fiber neuropathy: 10 cases and a literature review. Medicine (Baltimore) 2017;96:e6384.

14. Moon JY, Kim YC, Park MJ, et al. Cognitive assessment in complex regional pain syndrome patients. Korean J Pain 2009;22:28.

15. Tesio V, Torta DM, Colonna F, et al. Are fibromyalgia patients cognitively impaired? Objective and subjective neuropsychological evidence. Arthritis Care Res (Hoboken) 2015;67:143-50.

16. Romano R, Bertolino A, Gigante A, et al. Impaired cognitive functions in adult-onset primary cranial cervical dystonia. Parkinsonism Relat Disord 2014;20:162-5.

17. Weiner DK, Rudy TE, Morrow L, et al. The relationship between pain, neuropsychological performance, and physical function in community-dwelling older adults with chronic low back pain. Pain Med 2006;7:60-70.

18. Moseley GL, Zalucki N, Birklein F, et al. Thinking about movement hurts: the effect of motor imagery on pain and swelling in people with chronic arm pain. Arthritis Rheum 2008;59:623-31.

19. Koster-Hale J, Saxe R. Theory of mind: a neural prediction problem. Neuron 2013;79:836-48.

20. Shin NY, Kang DH, Jang JH, et al. Impaired recognition of social emotion in patients with complex regional pain syndrome. J Pain 2013;14:1304-9.

21. Sohn HS, Lee DH, Lee KJ, et al. Impaired empathic abilities among patients with complex regional pain syndrome (Type I). Psychiatry Investig 2016;13:34-42.

22. Subic-Wrana C, Beutel ME, Knebel A, et al. Theory of 
mind and emotional awareness deficits in patients with somatoform disorders. Psychosom Med 2010;72:404-11.

23. Stonnington CM, Locke DE, Hsu CH, et al. Somatization is associated with deficits in affective Theory of Mind. J

Psychosom Res 2013;74:479-85.

Cite this article as: Lee D, Kang DH. Suboptimal learning of tactile-spatial predictions in patients with complex regional pain syndrome. Ann Palliat Med 2020;9(6):4403-4407. doi: 10.21037/ apm-20-900 